\title{
Breastfeeding for oral health in preschool children (Protocol)
}

\author{
Arora A, Foster JP, Gillies D, Moxey AJ, Moody G, Curtis B
}

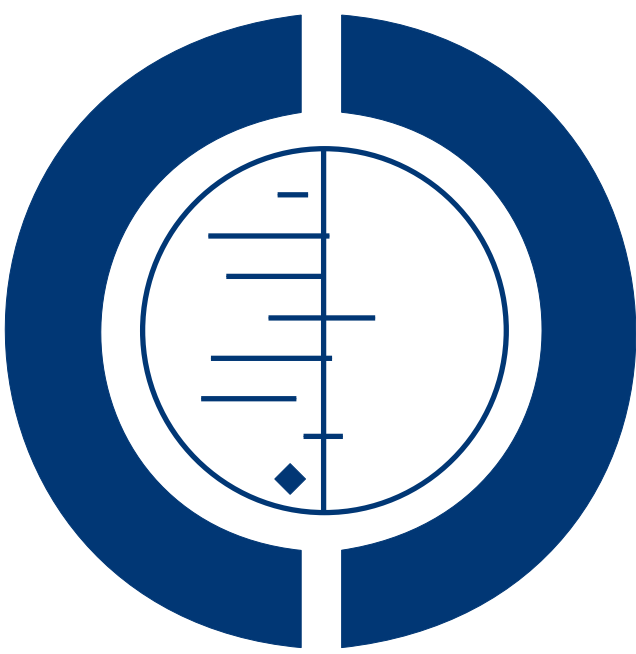

THE COCHRANE COLLABORATION $^{\circledR}$

This is a reprint of a Cochrane protocol, prepared and maintained by The Cochrane Collaboration and published in The Cochrane Library 2013, Issue 3

http://www.thecochranelibrary.com

\section{WILEY}


TABLE OF CONTENTS

HEADER . . . . . . . . . . . . . . . . . . . . . . . . . . . . . . . . . . . . 1

ABSTRACT . . . . . . . . . . . . . . . . . . . . . . . . . . . . . . . . . . . . . . 1

BACKGROUND . . . . . . . . . . . . . . . . . . . . . . . . . . . . . . . . . . . . . 2

OBJECTIVES . . . . . . . . . . . . . . . . . . . . . . . . . . . . . . . . . . . . . . .

METHODS . . . . . . . . . . . . . . . . . . . . . . . . . . . . . . . . . . . . . . 44

ACKNOWLEDGEMENTS . . . . . . . . . . . . . . . . . . . . . . . . . . . . . . . . . . . . . .

REFERENCES . . . . . . . . . . . . . . . . . . . . . . . . . . . . . . . . . . . . . . . . . . . . . . . . . . . . .

CONTRIBUTIONS OF AUTHORS . . . . . . . . . . . . . . . . . . . . . . . . . . . . . . . . . . . . . . 10

DECLARATIONS OF INTEREST . . . . . . . . . . . . . . . . . . . . . . . . . . . . . . . 10

SOURCES OF SUPPORT . . . . . . . . . . . . . . . . . . . . . . . . . . . . . . . . . . 10 


\title{
[Intervention Protocol]
}

\section{Breastfeeding for oral health in preschool children}

\author{
Amit Arora ${ }^{1,2,3}$, Jann P Foster ${ }^{4,5}$, Donna Gillies ${ }^{6}$, Annette J Moxey ${ }^{7}$, Gwen Moody $^{8}$, Bradley Curtis ${ }^{9}$ \\ ${ }^{1}$ Population Oral Health, Faculty of Dentistry, The University of Sydney, Westmead, Australia. ${ }^{2}$ Sydney Medical School, The University \\ of Sydney, Westmead, Australia. ${ }^{3}$ Sydney and Sydney South West Local Health District, Sydney, Australia. ${ }^{4}$ School of Nursing \& \\ Midwifery, University of Western Sydney, Sydney, Australia. ${ }^{5}$ Central Clinical School, Discipline of Obstetrics, Gynaecology and \\ Neonatology, Sydney Medical School/Sydney Nursing School, University of Sydney, Sydney, Australia. ${ }^{6}$ Western Sydney and Nepean \\ Blue Mountains Local Health Districts - Mental Health, Parramatta, Australia. ${ }^{7}$ Research Centre for Gender, Health \& Ageing, Faculty \\ of Health, University of Newcastle, Callaghan, Australia. ${ }^{8} \mathrm{CMC}$ Postnatal Service and Infant Care, Westmead Hospital, Wentworthville, \\ Australia. ${ }^{9}$ Eli Lily and Company, Carmel, Indianapolis, Indiana, USA
}

Contact address: Amit Arora, amit.arora@sydney.edu.au.

Editorial group: Cochrane Developmental, Psychosocial and Learning Problems Group.

Publication status and date: New, published in Issue 3, 2013.

Citation: Arora A, Foster JP, Gillies D, Moxey AJ, Moody G, Curtis B. Breastfeeding for oral health in preschool children. Cochrane Database of Systematic Reviews 2013, Issue 3. Art. No.: CD010416. DOI: 10.1002/14651858.CD010416.

Copyright (C) 2013 The Cochrane Collaboration. Published by John Wiley \& Sons, Ltd.

\begin{abstract}
A B S T R A C T
This is the protocol for a review and there is no abstract. The objectives are as follows:

Primary

- To compare the rates of dental caries in children who were bottle fed as infants to children who were breastfed up to six months, between 6 and 12 months, or longer
\end{abstract}

\section{Secondary}

- To compare the rates of dental caries in children who were: demand breastfed, non-demand breastfed

- To compare the rates of dental caries in children who were: breastfed via direct nursing, breastfed via a bottle, bottle fed with infant formula

- To compare the rates of dental caries in children who were: exclusively breastfed, partially breastfed, exclusively bottle fed with infant formula

- To compare the rates of dental caries in children who were: breastfed during day and night time, breastfed during the day only

This review examines the hypotheses that breastfed children would have lower rates of dental caries than bottle fed infants. 


\section{B A C K G R O U N D}

\section{Description of the condition}

Primary (baby) teeth begin to erupt around four to six months of age (Wake 2000; Arora 2012) and serve multiple roles such as chewing food, preserving space for permanent teeth and helping development of speech (Wake 2000; Arora 2012). Despite improvements in oral health, dental caries (decay) is identified as the most prevalent chronic disease of childhood (US Department of Health and Human Services 2000; Stecksen-Blicks 2004; Arora 2011a; Arora 2011b) and creates a major health burden both from a population perspective and for individual families who may have to deal with a young child suffering from toothache (Chu 1999; Arora 2011a).

Dental caries is a bacteriologically-mediated disease of multifactorial aetiology characterised by demineralisation of inorganic structures and destruction of the organic structures of the teeth (Richardson 1977; Addy 1986). The onset of dental caries has five necessary components: cariogenic bacteria (predominantly mutans streptococci), fermentable carbohydrates (the substrate), susceptible teeth (the host), lack of fluoride and time for the disease to develop (Gussy 2006; Fisher-Owens 2007). Cariogenic bacteria produce acids when they metabolise fermentable carbohydrates and demineralise the tooth structure.

Although the literature on the aetiology of dental caries is extensive, little is known about the aetiological pathways of dental caries in early childhood (Arora 2011b). This is highlighted by the fact that for the last 40 years, researchers have used different diagnostic criteria for dental caries in preschool children and refer to it by different names such as "baby bottle tooth decay", "nursing bottle syndrome" or "rampant caries" depending on the criteria used (Huntington 2002; Arora 2011b). International data on oral health of preschool children has noted wide variation depending on the definition used (Arora 2011b). In 1999, a workshop sponsored by the United States government addressed this issue and defined "early childhood caries" (ECC) as the presence of one or more decayed, missing (due to caries) or filled tooth surfaces on any primary tooth in children up to 71 months of age (Drury 1999), with "severe early childhood caries" (s-ECC) referring to one or more decayed, missing (due to caries) or filled tooth surfaces in the primary (baby) maxillary (upper) anterior (front) teeth (Drury 1999).

\section{Epidemiology}

There has been a decline in the prevalence of dental caries over the last 40 years, much of which occurred between 1973 and 1993, but over the last 20 years improvements have been at a slower rate (UK Department of Health 2006). In the United Kingdom, 48\% of five-year-olds had no dental decay in 1983 and this increased to 54\% in 1993 (UK Department of Health 2006). However, since then, successive national dental health surveys have shown little change in the prevalence of caries in five-year-old children (Lader 2004). In the USA, the National Health and Nutrition Examination Surveys tell a similar story. From 1988 to 1994 and 1999 to 2002 , there was no change in the prevalence of dental caries among preschool children (Beltran-Aguilar 2005). In Australia, the 2002 Child Dental Health Survey of Australia reported that the mean number of decayed, missing and filled teeth for five-year-olds was 1.83. Most alarmingly, it was reported that over $40 \%$ of five-yearolds had one or more decayed or missing teeth and $10 \%$ of those with caries were found to have more than seven decayed, missing or filled teeth (Armfield 2007).

Knowledge regarding the prevalence of ECC is currently limited in many other countries due to the lack of co-ordinated monitoring systems and difficulties faced in accessing this population. It has been reported that the prevalence of ECC ranges from $1 \%$ to 56\% (Aligne 2003; Montero 2003; Curzon 2004; van Palenstein Helderman 2006). However, its prevalence can be as high as $90 \%$ in disadvantaged families in developed countries and in developing countries (Nowak 2000; Mobley 2004).

\section{Consequences}

The early sign of dental caries presents as a "white spot lesion" along the gingival (gum) margin or on the occlusal (biting) surface of teeth coinciding with the distribution of dental plaque (bacteria). The interesting feature of this white spot lesion is that the loss of mineral occurs beneath the intact enamel surface. If the demineralisation process is not arrested, the carious lesion progresses to form a cavity, which may eventually lead to tooth fracture during chewing or biting.

ECC is a rapidly progressive disease that can be debilitating with short-term sequelae such as pain, infection, abscesses and chewing difficulty (Shepherd 1999; Arora 2011a). ECC has been associated with poor child growth and development, reduced general physical health, nutritional and sleep problems, and lasting psychological and psychosocial impediments including low selfesteem (Ripa 1988; Reisine 1998). Caries in young children are often managed by repeat prescriptions of antibiotics by general medical and dental practitioners and dental extractions under local or general anaesthetic (North 2007). This places a burden on the hospital services in most countries, and waiting lists for care can extend to over a year, with the majority on those waiting lists under five years of age (Bridgman 1999; Slack-Smith 2009).

Early childhood feeding practices can have a major influence on the development of dental caries in young children as mouths of young children have unique characteristics such as newly established bacterial flora and low resistance of newly erupted tooth surfaces to acid attack which accelerate the caries development process in young children (Vadiakas 2008). Our hypothesis is that breast-fed preschool children are less likely to develop dental caries than those that are bottle fed. 


\section{Description of the intervention}

Breastfeeding is one of the most important activities in infancy and early childhood. Human milk (breast milk) is uniquely engineered for human infants and is biologically the 'natural' way to feed infants. It is reported to be the primary source of nutrition for newborns before they are able to eat and digest other foods (Allen 2005). The documented benefits of breastfeeding to the mother include: increase in the levels of oxytocin resulting in less postpartum bleeding, accelerated weight loss and return to prepregnancy body weight, and reduced risk of ovarian and breast cancer (Kramer 2002; Allen 2005). Breast milk is reported to have numerous benefits for infants as it is uniquely suited to their needs throughout the period of lactation, providing nutrients required for at least the first six months (Kramer 2002; Allen 2005). Apart from providing ideal nutrition to the child, it has been suggested that it provides resistance to infectious diseases, enhances the immune system and reduces risk of chronic diseases and allergies (Kramer 2002; Allen 2005). There is some evidence that it reduces the incidence of gastroenteritis (Quigley 2007), respiratory infection (Quigley 2007), necrotising enterocolitis, otitis media, urinary tract infection, atopic disease, diabetes mellitus and obesity (Kramer 2002; Nommsen-Rivers 2003; Allen 2005; Harder 2005; Owen 2005). Breastfeeding's immunological protection is particularly important for vulnerable preterm and low birth weight infants (Allen 2005). Research also indicates a positive relationship between breastfeeding and improved cognitive development (Kramer 2002; Allen 2005). However, some longitudinal studies have reported that breast-fed children are more likely to have poor oral health outcomes such as dental caries (van Palenstein Helderman 2006; Yonezu 2006; Schluter 2007; Thitasomakul 2009). Therefore, the focus of this review is to review the impact of breastfeeding on the oral health of preschool children.

\section{How the intervention might work}

Components of human milk play a protective role. Immune factors such as secretory IgA and IgG can slow the growth of specific types of streptococcus mutans colonising the child's mouth (Eggert 1984; Oulis 1999; Prabhakar 2010). Lactoferrin in mothers' milk is also thought to have a bactericidal effect (destroying bacteria) (Palmer 2000). Furthermore, Erickson and colleagues evaluated the cariogenic potential of various liquids and reported that breast milk had a decay potential of 0.01 , close to water, which has a decay potential of 0.00 (Erickson 1998; Erickson 1999). Therefore, it may be reasonable to hypothesise that human milk is naturally protective to teeth. In addition, breast milk is not thought to be remaining stagnant (pooled) in the baby's mouth in the same way as bottle milk because the milk does not flow unless the baby is actively sucking (Erickson 1999). If babies are actively sucking then they are also swallowing, so pooling of breast milk in the mouth need not be considered a problem. Some consider pooling of the milk as an issue because it acts as a carbohydrate source for bacteria and could initiate the caries process. Furthermore, milk from the breast enters the baby's mouth behind the teeth. Conversely, bottle milk does not provide the protection of immunoglobulin's and contains high levels of dietary sugars (Arora 2011b).

\section{Why it is important to do this review}

The American Academy of Paediatrics and the Australian National Health and Medical Research Council suggest that breastfeeding should be continued until 12 months of age (NHMRC 2003; American Academy of Pediatrics 2005). The World Health Organization recommends breastfeeding should be continued until two years of age (WHO 1998). However, dental organisations such as the American Association of Pediatric Dentistry (AAPD) recommend that "ad libidum nocturnal breastfeeding should be avoided after the first primary tooth begins to erupt" (American Academy of Pediatric Dentistry 1996). This occurs around four to six months of age.

Some longitudinal studies have concluded that prolonged and exclusive breastfeeding does not contribute to the development of dental decay in preschool children (Oulis 1999; Kramer 2007), while other studies have found that breastfed children are more likely to have dental caries than bottle fed children (van Palenstein Helderman 2006; Yonezu 2006; Schluter 2007; Thitasomakul 2009). Of note, these studies were observational studies, due to ethical concerns about randomising mothers to a breastfeeding or bottle feeding conditions, and assessed the relationship of early childhood feeding practices and ECC. The dissemination of these contradictory messages remains problematic for both health care professionals and parents and therefore this systematic review will attempt to appraise and synthesise the evidence.

\section{O B J E C T I VES}

\section{Primary}

- To compare the rates of dental caries in children who were bottle fed as infants to children who were breastfed up to six months, between 6 and 12 months, or longer

\section{Secondary}

- To compare the rates of dental caries in children who were: demand breastfed, non-demand breastfed

- To compare the rates of dental caries in children who were: breastfed via direct nursing, breastfed via a bottle, bottle fed with infant formula 
- To compare the rates of dental caries in children who were: exclusively breastfed, partially breastfed, exclusively bottle fed with infant formula

- To compare the rates of dental caries in children who were: breastfed during day and night time, breastfed during the day only

This review examines the hypotheses that breastfed children would have lower rates of dental caries than bottle fed infants.

\section{METHODS}

\section{Criteria for considering studies for this review}

\section{Types of studies}

Intervention studies (randomised controlled trials (RCTs) and controlled clinical trials (CCTs)) and observational studies (cohort studies and case-control studies). Cross-sectional studies and single case reports will not be included.

\section{Types of participants}

Preschool children from birth up to six years ( $<72$ months) of age regardless of race, gender, socioeconomic status, health status or geographical location.

\section{Types of interventions}

The consumption of breast milk, via direct nursing or expression, compared with infant formula feeding.

\section{Types of outcome measures}

\section{Primary outcomes}

Rates of dental caries in the primary dentition. Dental caries will be measured using the decayed/missing/filled teeth (DMFT; number of decayed, missing due to caries or filled teeth) or decayed, missing, or filled surface (DMFS; number of decayed, missing due to caries, or filled tooth surfaces) scores.

\section{Secondary outcomes}

\section{Child outcomes}

- Emergency visits to healthcare provider due to dental problems

- Prescriptions for antibiotics for dental infections

- Prescriptions for analgesics for dental pain

\section{Other outcomes}

- Parental work days lost because of emergency dental visits or follow-up care

- Financial cost

\section{Search methods for identification of studies}

\section{Electronic searches}

We will search for potential RCTs, CCTs, cohort studies and casecontrol studies in the following databases.

- Cochrane Central Register of Controlled Trials

(CENTRAL) (The Cochrane Library).

- MEDLINE

- EMBASE

- PubMed

- Cochrane Oral Health Group's Specialised Register

- CINAHL

- Social Science Citation Index

- Conference Proceedings Index

- Dissertation Abstracts

Detailed search strategies for each of the databases will be based on the following search strategy for MEDLINE.

1. Breast Feeding/

2. (breastfeed $\$$ or breastfed or breast-feed $\$$ or breast-fed).tw.

3. Milk, Human/

4. (breastmilk\$ or breast-milk\$ or human milk\$).tw.

5. or/1-4

6. exp Dental Caries/

7. Dental Caries Susceptibility/

8. Tooth Demineralization/

9. (caries\$ or cario $\$$ ).tw.

10. oral health/

11. oral health.tw.

12. dental health.tw.

13. (dental adj5 (cavit\$ or decay $\$$ or lesion $\$$ or deminerali $\$$ or reminerali\$)).tw.

14. (tooth adj5 (cavit\$ or decay\$ or lesion $\$$ or deminerali $\$$ or reminerali\$)).tw.

15. (teeth adj5 (cavit\$ or decay $\$$ or lesion $\$$ or deminerali $\$$ or reminerali\$)).tw.

16. ECC.tw.

17. or/6-15

18. 5 and 17

The MEDLINE strategy will be adapted for other databases, taking account the indexing terms and syntax of each database searched. 


\section{Searching other resources}

We will check reference lists of articles for further studies. Where possible, we will contact the first author of included studies to assist in identifying relevant published and unpublished studies. We will seek translation of abstracts of articles that are not in English to determine whether they fit the inclusion criteria. If they do, we will ask the translator to perform data extraction.

\section{Handsearching}

We will handsearch some key journals in this field (from 1990) such as Community Dental Health, International Journal of Paediatric Dentistry, Journal of Public Health Dentistry, Community Dentistry and Oral Epidemiology, Pediatrics, Pediatric Dentistry to identify articles that may have been missed in database and reference list searches. This will also help us identify very recent publications, which have yet to be cited by other publications or included on the electronic databases.

\section{Searching the grey literature}

We will search for grey literature in OpenSIGLE (System for Information on Grey Literature). We will search for dissertations and theses in CINAHL and Dissertation Abstracts databases, Networked Digital Library of Theses and Dissertations (in United States), Australasian Digital Theses Program, and DART-Europe E-theses Portal (in Europe).

We will search for conference proceedings in the Index to Scientific and Technical Proceedings the Conference Papers Index and in the catalogues of libraries such as the British Library and large research libraries. The abstracts in conference proceedings may present limited information and there may be differences between data presented in abstracts and final reports. If we find a relevant abstract, we will contact the authors to obtain the full report.

\section{Reference lists}

We will search the reference lists of the studies included in this review and relevant papers to identify additional studies in the published or unpublished literature.

\section{Data collection and analysis}

The review will be conducted using the Cochrane Collaboration's statistical software, Review Manager (Review Manager 2012).

\section{Selection of studies}

Two authors (AA and JPF) will independently assess for inclusion all the potential studies identified as a result of the search strategy. We will obtain the full text of papers or reports for studies that appear relevant or for which more information is needed to determine relevance and the two authors will independently screen these to determine whether they meet the criteria for inclusion. We will resolve disagreement about eligibility through discussion and, when disagreements cannot be resolved, will seek advice from the other authors (BC and AJM). We will seek additional information from the authors of the studies as necessary to resolve questions about the relevance or methodology. We will record the reasons for excluding any studies (for example, single case reports) where it is not clear from the title or abstract in search databases. None of the authors will be blind to the authors, institutions, or the journals of publication of the articles. We will seek translation of abstracts of articles that are not in English to determine whether they are of interest. If they are, we will arrange for translation of the full text.

Specifically, we will:

1. merge search results using reference management software and remove duplicate records of the same report;

2. examine titles and abstracts to remove irrelevant reports;

3. retrieve full text of the potentially relevant reports;

4. link together multiple reports of the same study;

5. examine full text reports for compliance of studies with eligibility criteria;

6. correspond with investigators, when appropriate, to clarify study eligibility;

7. at all stages, note reasons for inclusion and exclusion of articles, resolving disagreements through consensus;

8. make final decisions on study inclusion and proceed to data collection; and

9. resolve all discrepancies through a process of consensus.

\section{Data extraction and management}

Four authors (AA, DG, JPF, GM) will independently extract data from the full-text articles using a specifically designed spread sheet to manage the information. We will resolve discrepancies through discussion or, if required, we will consult a review arbiter (BHC). We will enter data into Review Manager 2012 and check them for accuracy. When information regarding any of the above is missing or unclear, we will attempt to contact authors of the original reports to provide further details. We will collect data according to the following sequence and will include recording of the following information.

1. General information - published/unpublished, author, title, year research completed and year research published, journal, ethics and consent process, country, language, funding source, conflict of interest, contact address.

2. Study characteristics - study design, research objective, sample size, study duration, inclusion and exclusion criteria. In addition, for RCTs and CCTs, we will record the method of allocation, allocation concealment and blinding.

3. Participant characteristics - country, setting (including fluoridation and socioeconomic status), age, gender, medical factors. In addition, for RCTs and CCTs, we will record the number of participants recruited in each group and number of 
participants on follow-up. For cohort studies, we will record the number of participants at the start and the end of the study and for case-control studies, we will record the number of cases and the number of controls.

4. Intervention characteristics or exposures - breastfeeding duration and frequency.

5. Outcome characteristics - primary and secondary outcome measures.

6. Results and conclusions - what was found and what conclusions have been drawn from the study.

7. Other factors such as diet in preschool children.

\section{Assessment of risk of bias in included studies}

At least two authors (AA and JPF) will independently assess the risk of bias of included studies, resolving any disagreements by discussion with a third author (DG).

We will use the following criteria from the Cochrane Handbook for Systematic Reviews of Interventions to assess the risk of bias in the included studies (Higgins 2011).

1. Sequence generation: was the allocation sequence adequately generated?

2. Allocation concealment: was allocation adequately concealed?

3. Blinding of participants, personnel and outcome assessors for each main outcome or class of outcomes: was knowledge of the allocated intervention adequately prevented during the study?

4. Incomplete outcome data for each main outcome or class of outcomes: were incomplete data adequately addressed?

5. Selective outcome reporting: are reports of the study free of suggestion of selective outcome reporting?

6. Other sources of bias: was the study apparently free of other problems that could put it at a high risk of bias; for example, evidence of baseline differences, confounding or differential loss to follow-up; differing measures of exposure and outcomes in cohort and case control studies; and inappropriate control groups in case control studies?

We will use the Cochrane Risk of Bias tool for all studies in the first instance (Higgins 2011). If required, we will assss the risk of bias for observational studies using the NewCastle Ottawa Scale (Wells 2004).

We will assess each trial for risk of bias based on the criteria listed above and mark as:

- low risk of bias;

- unclear risk of bias; or

- high risk of bias.

We will resolve any discrepancies by mutual discussion and consensus.

\section{Measures of treatment effect}

\section{Dichotomous data}

For dichotomous data, we will present results as risk ratios (RRs) with $95 \%$ confidence intervals (CIs). If there is a statistically significant difference, we will report risk differences (RDs) and calculate the number needed to treat for additional benefit (NNTB) or number needed to treat for an additional harmful outcome $(\mathrm{NNH})$, and associated $95 \%$ CIs.

\section{Continuous data}

For continuous data, we will use the mean difference (MD) to pool data across studies if outcomes are reported using the same measure. We will use the standardised mean difference (SMD) to combine trials that measure the same outcome, but use different measures.

\section{Unit of analysis issues}

We will include cluster-randomised trials in the analyses along with individually randomised trials. We will correct the standard deviation for cluster-randomised controlled trials using an estimate of the intracluster correlation coefficient (ICC) derived from the trial (if possible), or from another source (Higgins 2011). If ICCs from other sources are used, we will report this and conduct sensitivity analyses to investigate the effect of including clusterrandomised trials.

\section{Dealing with missing data}

Where necessary, we will contact the authors of the included studies to supply any unreported data (for example groups means and standard deviations (SDs), details of dropouts, details of any interventions).

If a study reports outcomes only for participants completing the study or only for participants who followed the protocol, we will contact the authors and ask them to provide additional information to permit analyses according to an intention-to-treat analysis. We will describe missing data and drop-outs/attrition for each included study in the 'Risk of Bias' tables, and discuss the extent to which these could alter the results/conclusion of the review. We will report the number of participants who were included in the final analysis as a proportion of all participants in each study. We will provide reasons for missing data in the narrative summary. We will assess the sensitivity of any primary meta-analyses to missing data using the strategy recommended by Higgins 2011 .

\section{Assessment of heterogeneity}

We will assess clinical heterogeneity (for example, participant characteristics, study settings, treatment method) by examining the relevant criteria. If considerable clinical heterogeneity is observed, we will conduct subgroup analyses to explore their effects. We will use $\mathrm{Chi}^{2}$ test and $\mathrm{I}^{2}$ analysis to evaluate heterogeneity (Higgins 
2002). For statistical heterogeneity we will interpret a $P$ value of less than 0.10 for the $\mathrm{Chi}^{2}$ test of heterogeneity and/or an $\mathrm{I}^{2}$ value of between $50 \%$ and $100 \%$ (indicating substantial or considerable heterogeneity). If there is evidence of substantial or considerable heterogeneity, we will use a random-effects model for meta-analysis.

\section{Assessment of reporting biases}

To identify whether there has been selective reporting of data, we will try to obtain the study protocols of all included studies and we will compare outcomes reported in the protocol to those reported in the findings for each of the included studies.

We will draw funnel plots (estimated differences in treatment effects against their standard error) if we find sufficient studies (Higgins 2011). Asymmetry might be due to publication bias or to systematic differences between small and large studies. If a relationship is identified, we will further examine the clinical diversity of the studies to provide a possible explanation. As a direct test for publication bias, we will compare results extracted from published journal reports with results obtained from other sources (including correspondence).

\section{Data synthesis}

The results of the studies will be analysed using the statistical package using Review Manager 2012. Data will be summarised in a meta-analysis if they are sufficiently homogeneous, both clinically and statistically.

We plan to perform statistical analysis according to the recommendations of the Cochrane Collaboration (Higgins 2011). We plan to analyse all infants randomised on an ITT basis. We plan to analyse treatment effects in the individual trials and plan to use a fixed-effect model for meta-analysis in the first instance to combine the data. Where substantial heterogeneity exists, the potential cause of heterogeneity will be examined in subgroup and sensitivity analysis. When we judge meta-analysis to be inappropriate, we plan to analyse and interpret individual trials separately. For estimates of typical relative risk and risk difference, we will use the Mantel-Haenszel method. For measured quantities, we used the inverse variance method.
If outcome data are reported as a median or range, or as a mean without a variance, we will report these data in additional tables.

\section{Subgroup analysis and investigation of heterogeneity}

We will discuss possible reasons for heterogeneity and conduct subgroup analyses accordingly, where data permit. We will carry out subgroup analyses to explore the following aspects.

- Total duration of breastfeeding (<6 months; 6 to 12 months; > 12 months).

- Demand breastfeeding versus non-demand breastfeeding.

- Method of feeding (breastfed via direct nursing; breastfed via a bottle; bottle fed with infant formula).

- Type of feeding (exclusively breastfed, partially breastfed, exclusively bottle fed with infant formula).

- Socioeconomic status.

- Fluoridation status.

\section{Sensitivity analysis}

We will conduct sensitivity analyses to determine whether findings are sensitive to restricting the analyses to studies judged to be at low risk of bias. In these analyses, we will restrict the analysis to: (a) only studies with a low risk of selection bias (associated with sequence generation or allocation concealment); (b) only studies with low risk of performance bias (associated with issues of blinding); (c) only studies with low risk of attrition bias (associated with completeness of data). In addition, we will assess the sensitivity of findings to any imputed data.

\section{ACK NOW LEDGEMENTS}

The authors would like to thank Mr Jeremy Cullis, Senior Librarian, University of Sydney and Ms Margaret Anderson, Trials Search Co-ordinator of Cochrane Developmental, Psychosocial and Learning Problems Group for development of the search strategy. The authors would also like to thank Professor Geraldine Macdonald and Ms Laura MacDonald of the Cochrane Developmental, Psychosocial and Learning Problems Group and Dr Md. Bayzidur Rahman, University of New South Wales, for their advice and support. 


\section{R E F E R E N C E S}

\section{Additional references}

\section{Addy 1986}

Addy M, Dummer PM, Griffiths G, Hicks R, Kingdom A, Shaw WC. Prevalence of plaque, gingivitis and caries in 1112-year-old children in South Wales. Community Dentistry and Oral Epidemiology 1986;14(2):115-8.

Aligne 2003

Aligne CA, Moss ME, Auinger P, Weitzman M. Association of pediatric dental caries with passive smoking. JAMA 2003;289(10):1258-64.

Allen 2005

Allen J, Hector D. Benefits of breastfeeding. New South Wales Public Health Bulletin 2005;16(3-4):42-6.

American Academy of Pediatric Dentistry 1996 American Association of Pediatric Dentistry. Oral health policies - reference manual. Pediatric Dentistry 1996;18(6): 24-9.

American Academy of Pediatrics 2005

American Academy of Pediatrics. Breastfeeding and the use of human milk. Pediatrics 2005;115(2):494-506.

Armfield 2007

Armfield JM, Slade GD, Spencer AJ. Water Fluoridation and Children's Oral Health. The Child Dental Health Survey, Australia, 2002. Dental Statistics and Research Series No.36. Canberra, ACT: Australian Institute of Health and Welfare, 2007.

Arora 2011a

Arora A, Scott JA, Bhole S, Do L, Schwarz E, Blinkhorn AS. Early childhood feeding practices and dental caries in preschool children: a multi-centre birth cohort study. $B M C$ Public Health 2011;11:28.

Arora 2011b

Arora A, Schwarz E, Blinkhorn AS. Risk factors for early childhood caries in disadvantaged populations. Journal of Investigative and Clinical Dentistry 2011;2:1-6.

Arora 2012

Arora A, Mistry D. Perceptions of Australian mothers on infant teething: a pilot investigation using a mixed methods approach. Social Science and Dentistry 2012;2(1):3-9.

Beltran-Aguilar 2005

Beltran-Aguilar ED, Barker LK, Canto MT, Dye BA, Gooch BF, Griffin SO, et al.Surveillance for dental caries, dental sealants, tooth retention, edentulism and enamel fluorosis in United States, 19881-994 and 1999-2002. MMWR Surveillance Summaries 2005;54(3):1-44.

Bridgman 1999

Bridgman CM, Ashby D, Holloway PJ. An investigation of the effects on children of tooth extraction under general anaesthesia in general dental practice. British Dental Journal 1999;186(5):245-7.
Chu 1999

Chu CH, Fung DSH, Lo CM. Dental caries status of preschool children in Hong Kong. British Dental Journal 1999;187(11):616-20.

Curzon 2004

Curzon MEJ, Preston AJ. Risk groups: nursing bottle caries/caries in the elderly. Caries Research 2004;38(suppl 1):24-33.

Drury 1999

Drury TF, Horowitz AM, Ismail AI, Maertens MP, Rozier RG, Selwitz RH. Diagnosing and reporting early childhood caries for research purposes. A report of a workshop sponsored by the National Institute of Dental and Craniofacial Research, the Health Resources and Services Administration, and the Health Care Financing Administration. Journal of Public Health Dentistry 1999;59 (3):192-7.

\section{Eggert 1984}

Eggert FM, Gurner BW. Reaction of human colostral and early milk antibodies with oral streptococci. Infection and Immunity 1984;44(3):660-4.

Erickson 1998

Erickson PR, McClintock KL, Green N, LaFleur J. Estimation of the caries-related risk associated with infant formulas. Pediatric Dentistry 1998;20(7):395-403.

\section{Erickson 1999}

Erickson PR, Mazhari E. Investigation of the role of human breast milk in caries development. Pediatric Dentistry 1999; 21(2):86-90.

\section{Fisher-Owens 2007}

Fisher-Owens SA, Gansky S, Platt L, Weintraub J, Soobader $\mathrm{M}$, Bramlett $\mathrm{M}$, et al.Influences on children's oral health: a conceptual model. Pediatrics 2007;120(3):e510-20.

Gussy 2006

Gussy MG, Waters EG, Walsh O, Kilpatrick NM. Early childhood caries: current evidence for aetiology and prevention. Journal of Paediatrics and Child Health 2006;42 (1-2):37-43.

\section{Harder 2005}

Harder T, Bergmann R, Kallischnigg G, Plagemann A. Duration of breastfeeding and risk of overweight: a metaanalysis. American Journal of Epidemiology 2005;162(5): 397-403.

Higgins 2002

Higgins JPT, Thompson SG. Quantifying heterogeneity in a meta-analysis. Statistics in Medicine 2002;21(11):1539-58.

\section{Higgins 2011}

Higgins JPT, Green S (editors). Cochrane Handbook for Systematic Reviews of Interventions. Version 5.1.0 [updated March 2011]. The Cochrane Collaboration, 2011. Available from: www.cochrane-handbook.org. 


\section{Huntington 2002}

Huntington NL, Kim IJ, Hughes CV. Caries-risk factors for Hispanic children affected by early childhood caries. Pediatric Dentistry 2002;24(6):536-42.

\section{Kramer 2002}

Kramer MS, Kakuma R. Optimal duration of exclusive breastfeeding. Cochrane Database of Systematic Reviews 2002, Issue 1. [DOI: 10.1002/14651858.CD003517]

\section{Kramer 2007}

Kramer MS, Vanilovich I, Matush L, Bogdanovich N, Zhang X, Shishko G, et al.The effect of prolonged and exclusive breast-feeding on dental caries in early schoolage children. New evidence from a large randomized trial. Caries Research 2007;41(6):484-8.

\section{Lader 2004}

Lader D, Chadwick B, Chestnutt L, Harker R, Morris J, Nuttal N, et al.Childrens Dental Health in the United Kingdom, 2003. London: Office for National Statistics, 2004.

\section{Mobley 2004}

Mobley CC. Lifestyle interventions for "diabesity": the state of the science. Compendium of Continuing Education in Dentistry 2004;25(3):207-18.

\section{Montero 2003}

Montero MK, Douglass JM, Mathieu GM. Prevalence of dental caries and enamel defects in Connecticut Head Start children. Pediatric Dentistry 2003;25(3):235-9.

\section{NHMRC 2003}

National Health and Medical Research Council. Dietary Guidelines for Children and Adolescents in Australia. Canberra, ACT: National Health and Medical Research Council, 2003.

Nommsen-Rivers 2003

Nommsen-Rivers L. The long-term effects of early nutrition: the role of breastfeeding on cholesterol levels. Journal of Human Lactation 2003;19(1):103-4.

\section{North 2007}

North S, Davidson LE, Blinkhorn AS, Mackie IC. The effects of a long wait for childrens dental general anaesthesia. International Journal of Paediatric Dentistry 2007;17(2): $105-9$.

\section{Nowak 2000}

Nowak AJ, Warren JJ. Infant oral health and oral habits. Pediatric Clinics of North America 2000;47(5):1043-66.

\section{Oulis 1999}

Oulis CJ, Berdouses ED, Vadiakas G, Lygidakis NA. Feeding practices of Greek children with and without nursing caries. Pediatric Dentistry 1999;21(7):409-16.

Owen 2005

Owen CG, Martin RM, Whincup PH, Smith GD, Cook DG. Effect of infant feeding on the risk of obesity across the life course: a quantitative review of published evidence. Pediatrics 2005;115(5):1367-77.
Palmer 2000

Palmer B. Breastfeeding and infant caries: no connection. ABM News and Views 2000;6(4):27-31.

\section{Prabhakar 2010}

Prabhakar AR, Kurthukoti AJ, Gupta P. Cariogenicity and acidogenicity of human milk, plain and sweetened bovine milk: an in vitro study. Journal of Clinical Pediatric Dentistry 2010;34(3):239-47.

\section{Quigley 2007}

Quigley MA, Kelly YJ, Sacker A. Breastfeeding and hospitalization for diarrheal and respiratory infection in the United Kingdom Millennium Cohort Study. Pediatrics 2007;119(4):e837-42.

\section{Reisine 1998}

Reisine S, Douglass JM. Psychosocial and behavioral issues in early childhood caries. Community Dentistry and Oral Epidemiology 1998;26(1 suppl):32-44.

\section{Review Manager 2012}

The Nordic Cochrane Centre, The Cochrane Collaboration. Review Manager (RevMan). 5.2. Copenhagen: The Nordic Cochrane Centre, The Cochrane Collaboration, 2012.

\section{Richardson 1977}

Richardson AS, Boyd MA, Conry RF. A correlation study of diet, oral hygiene and dental caries in 457 Canadian children. Community Dentistry and Oral Epidemiology 1977; 5(5):227-30.

\section{Ripa 1988}

Ripa LW. Nursing caries: a comprehensive review. Pediatric Dentistry 1988;10(4):268-82.

\section{Schluter 2007}

Schluter PJ, Durward C, Cartwright S, Paterson J. Maternal self-report of oral health in 4-year-old Pacific children from South Auckland, New Zealand: findings from the Pacific Islands Families Study. Journal of Public Health Dentistry 2007;67(2):69-77.

\section{Shepherd 1999}

Shepherd MA, Nadanovsky P, Sheiham A. The prevalence and impact of dental pain in 8-year old school children in Harrow, England. British Dental Journal 1999;187(1): $38-41$.

Slack-Smith 2009

Slack-Smith L, Colvin L, Leonard H, Kilpatrick N, Bower C, Brearley Messer L. Factors associated with dental admissions for children aged under 5 years in Western Australia. Archives of Disease in Childhood 2009;94(7): 517-23.

\section{Stecksen-Blicks 2004}

Stecksen-Blicks C, Sunnegardh K, Borssen E. Caries experience and background factors in 4-year-old children: time trends 1967-2002. Caries Research 2004;38(2): $149-55$.

\section{Thitasomakul 2009}

Thitasomakul S, Piwat S, Thearmontree A, Chankanka O, Pithpornchaiyakul W, Madyusoh S. Risks for early 
childhood caries analyzed by negative binomial models.

Journal of Dental Research 2009;88(2):137-41.

UK Department of Health 2006

Department of Health. Information about dental public health in the NHS, Improvements in oral health over the past 30 years, the Government's Oral Health Plan for England, and Guidance for Strategic Health Authorities on Water Fluoridation. London: Department of Health, 2006.

US Department of Health and Human Services 2000

United States Department of Health and Human Services. Oral Health in America - A Report of the Surgeon General (Executive Summary). Rockville, MD: US Department of Health and Human Services, National Institute of Dental and Craniofacial Research, National Institutes of Health, 2000

Vadiakas 2008

Vadiakas G. Case definition, aetiology and risk assessment of early childhood caries (ECC): a revisited review. European Archives of Paediatric Dentistry 2008;9(3):114-25.

van Palenstein Helderman 2006

van Palenstein Helderman WH, Soe W, van't Hof MA.
Risk factors of early childhood caries in a southeast Asian population. Journal Dental Research 2006;85(1):85-8.

Wake 2000

Wake M, Hesketh K, Lucas J. Teething and tooth eruption in infants: a cohort study. Pediatrics 2000;106(6):1374-9.

Wells 2004

Wells GA, Shea B, O'Connell D, Peterson J, Welch V, Losos $\mathrm{M}$, et al.The NewCastle Ottawa Scale (NOS) for assessing the quality of nonrandomised studies in a meta analyses. http://www.ohri.ca/programs/clinical_epidemiology/ oxford.asp (accessed 20 January 2013).

WHO 1998

World Health Organization. Evidence for the Ten Steps to Successful Breastfeeding. Geneva: World Health Organization, 1998.

Yonezu 2006

Yonezu T, Ushida N, Yakushiji M. Longitudinal study of prolonged breast or bottle-feeding on dental caries in Japanese children. Bulletin of Tokyo Dental College 2006;47 (4):157-60.

* Indicates the major publication for the study

\section{CONTRIBUTIONSOFAUTHORS}

All authors drafted the protocol and agreed on the final text.

\section{DECLARATIONSOF INTEREST}

Amit Arora - none known

Jann P Foster - none known

Donna Gillies - none known

Annette J Moxey - none known

Gwen Moody- none known

Bradley Curtis - none known

\section{SOURCES OF SUPPORT}




\section{Internal sources}

- Oral Health Foundation, University of Sydney, Australia.

\section{External sources}

- Australian National Health and Medical Research Council (Project Grant - 1033213), Australia.

- Centre for Oral Health Strategy, NSW Health, Australia. 